\title{
Cervical Intramedullary Epidermoid Cyst with Liquid Contents
}

\author{
Amit Agarwal', Arvind Bhake ${ }^{2}$, Anand Kakani ${ }^{1}$ \\ ${ }^{1}$ Department of Neurosurgery, Datta Meghe Institute of Medical Sciences, Wardha, Maharashtra, India \\ ${ }^{2}$ Department of Pathology, Datta Meghe Institute of Medical Sciences, Wardha, Maharashtra, India
}

\begin{abstract}
Intramedullary spinal epidermoid cysts are benign ectopic embryological growths with reported incidence of less than $1 \%$ of intramedullary tumors. In this case we report an unusual cervical intramedullary epidermid with liquid contents. A 40year-old patient presented with progressive weakness of all four limbs of four months duration, bowel and bladder disturbances of two days duration, pain and paresthesias in all four limbs. Magnetic resonance imaging (MRI) revealed a well defined intramedullary lesion extending from $\mathrm{C} 2-\mathrm{C} 3$ level with widening of the cord. The lesion was hypointense on T1W images, hyperintense on $\mathrm{T} 2 \mathrm{~W}$ and fluid attenuation and inversion recovery images with thin rim of enhancement after contrast administration. Histopathological examination of the excised specimen revealed epidermal lining and keratinous material features of an epidermoid cyst. As in present case, rarely epidermoid cyst can have clear contents, and an MRI finding can closely mimic the features of arachnoid cyst, findings not classical and is different than described in literature.
\end{abstract}

Key Words: Intramedullary epidermoid, Magnetic resonance imaging, Epidermal cyst, Spinal cord neoplasms

\section{Introduction}

Intramedullary spinal epidermoid cysts are benign ectopic embryological growths with reported incidence of less than $1 \%$ of intramedullary spinal cord tumors [1] and out of these intramedullary epidrmoid lesions within the cervical spinal cord are further rarer [1,2]. We report an unusual case of cervical intramedullary epidermoid with clear contents that was managed successfully.

\section{Case Report}

A 40-year-old female presented with progressive weakness of all four limbs of four months duration, bowel and bladder disturbances of two days duration. She was also complaining of pain and paresthesias in all four limbs. The patient was presently bedridden. There was decreased sen- sation to pain, touch, temperature and posterior column sensations below neck. There was no history of trauma and fever. On physical examination there was no dermal sinus or other stigmata. Higher mental functions and cranial nerve examination was normal. Motor examination revealed increased tone in all four limbs, muscle bulk was normal in all groups. Power was grade 4/5 in all groups. Deep tendon reflexes were exaggerated in both upper and lower limbs with bilateral Hoffmann and Babinski signs. Sensory examination revealed decreased sensation to all modalities below C5 level. Magnetic resonance imaging (MRI) revealed a well defined intramedullary lesion extending from $\mathrm{C} 2-\mathrm{C} 3$ level with widening of the cord. The lesion was hypointense on T1W images, hyperintense on $\mathrm{T} 2 \mathrm{~W}$ and fluid attenuation and inversion recovery images with thin rim of enhancement after contrast administration (Figs. 1 and 2). The patient underwent laminectomy from $\mathrm{C} 1$ to $\mathrm{C} 4$. During surgery after opening the dura, pale and widened cord could

Received Jun 23, 2010; 1st Revised Jul 12, 2010; Accepted Aug 10, 2010

Corresponding author: Amit Agrawal, MCh

Department of Neurosurgery, Datta Meghe Institute of Medical Sciences, Sawangi (Meghe), Wardha 442004, Maharashtra, India

Tel: +91-7152-287701, Fax: +91-7152-287719, E-mail: dramitagrawal@gmail.com,dramit_in@yahoo.com 

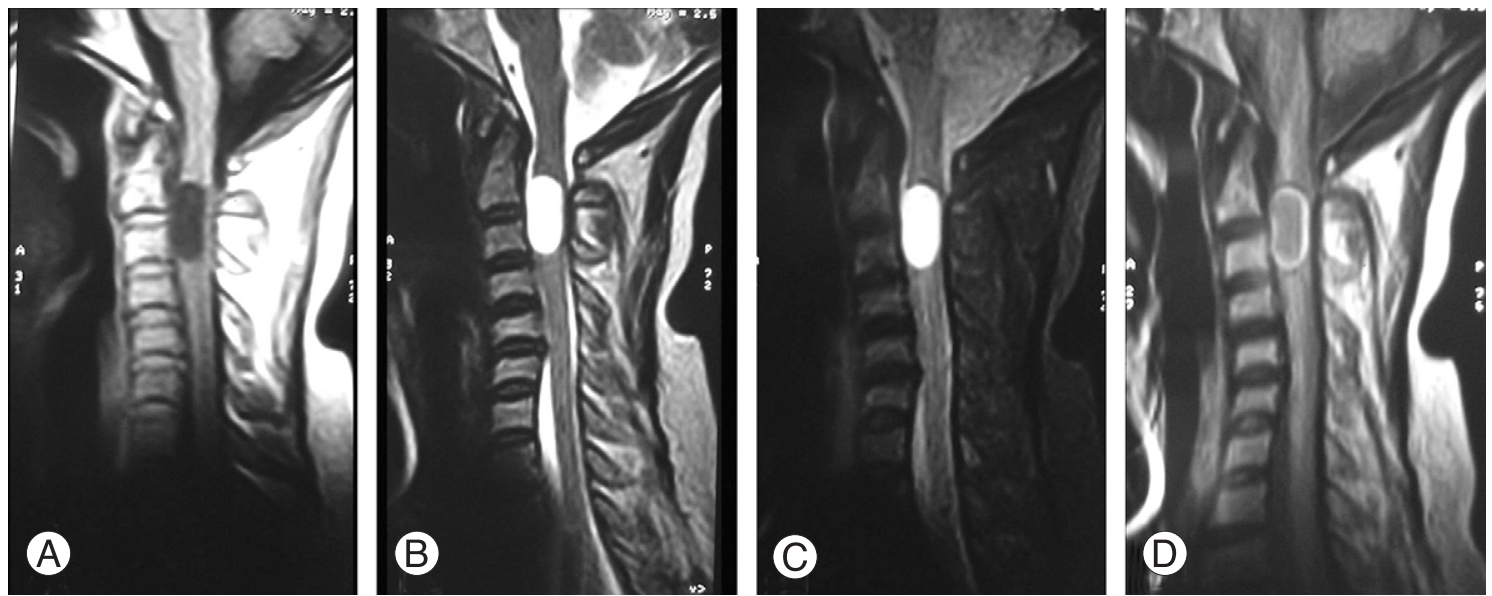

Fig. 1. Preoperative MRI images demonstrating a well define cervical intramedullary lesion on hypointense T1W (A), becoming hyperintnese on T2W (B) and fluid attenuation and inversion recovery (C) images with thin rim of enhancement (D). MRI: Magnetic resonance imaging.

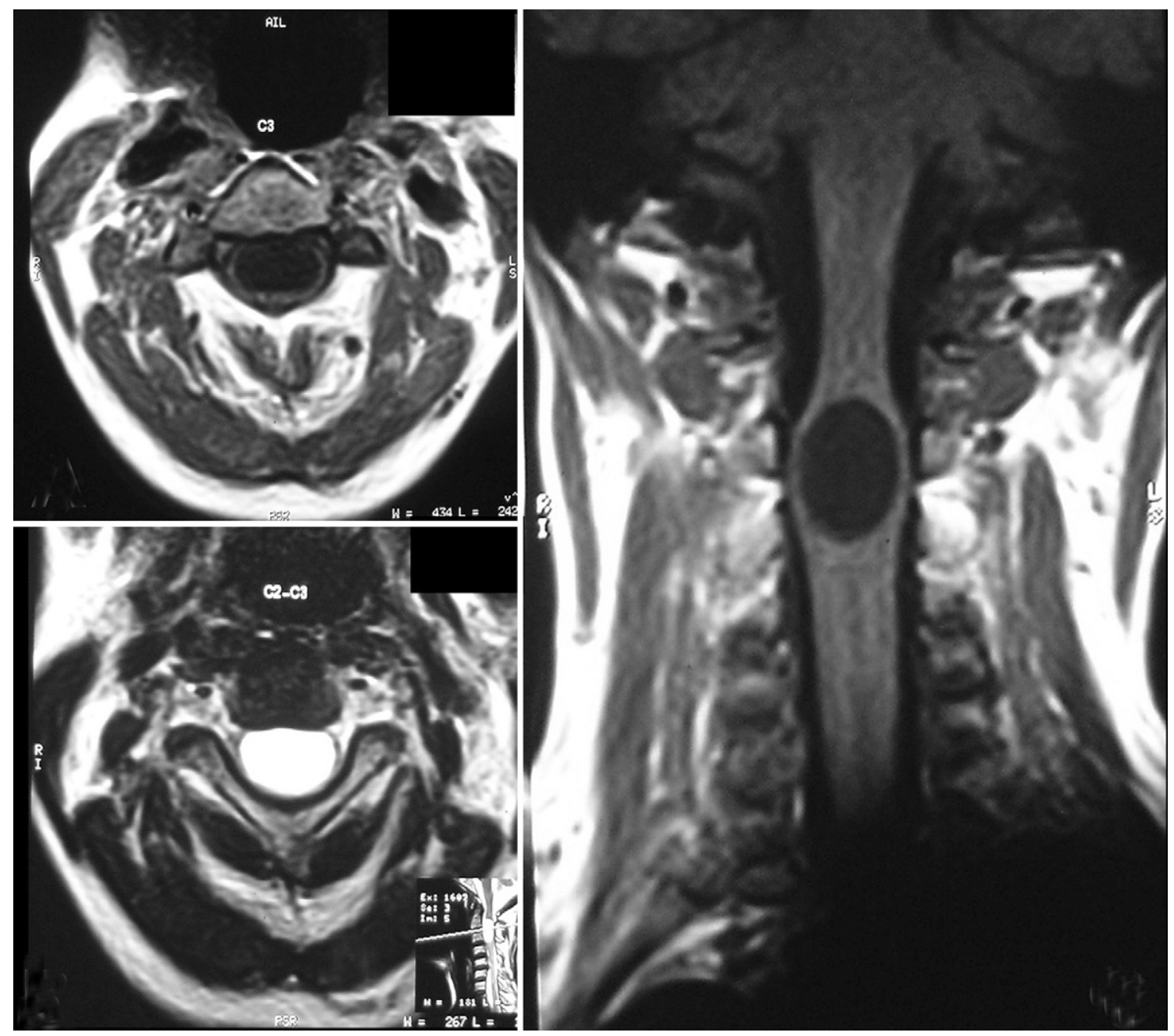

Fig. 2. Magnetic resonance imaging $\mathrm{T} 1 \mathrm{~W}$ and $\mathrm{T} 2 \mathrm{~W}$ axial images and $\mathrm{T} 1 \mathrm{~W}$ coronal images showing further details of the lesion. 
be seen. A midline myelotomy was performed and a thin walled cyst with pale yellow clear contents was recognized. There was ill defined capsule, difficult to separate from the surrounding cord; only small fragment for biopsy could be taken (Fig. 3). Histopathological examination of the excised specimen revealed features of an epidermoid cyst (Fig. 4). Immediately after surgery in post-operative period the patient deteriorated in motor power but recovered to the pre-operative period over a period of one week. At the 6 month follow up she reported improvement in motor power with reduction in the spasticity, complete control in bowel and bladder functions and able to carry her activities of daily living. Follow up MRI showed complete excision of the lesion and thinning of the cord at that level (Fig. 5).
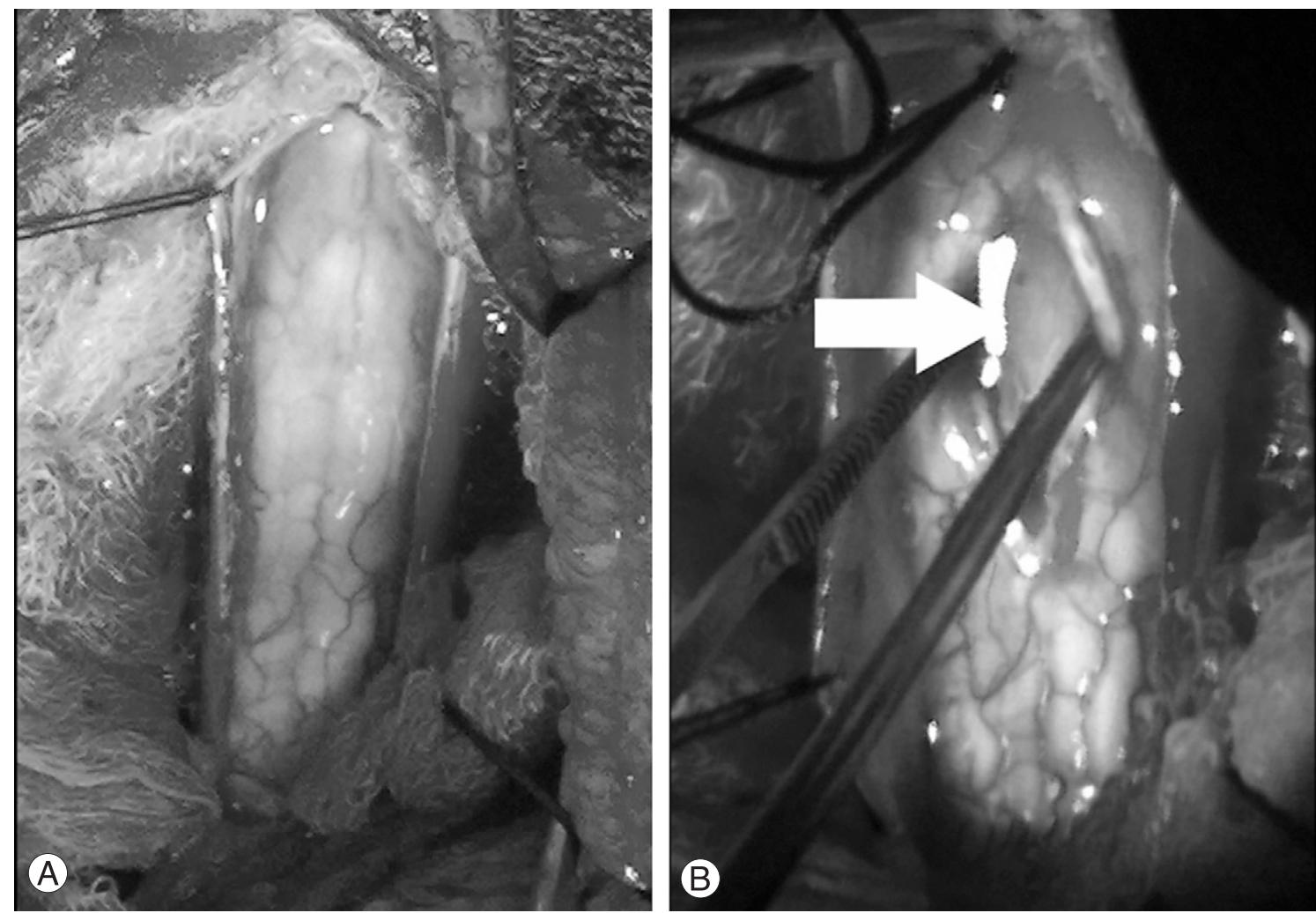

Fig. 3. Intra-operative photographs (A) showing widened and pale cord, (B) after opening the cord and cyst capsule clear contents of the cyst cavity (ignore the artifact next to arrow).
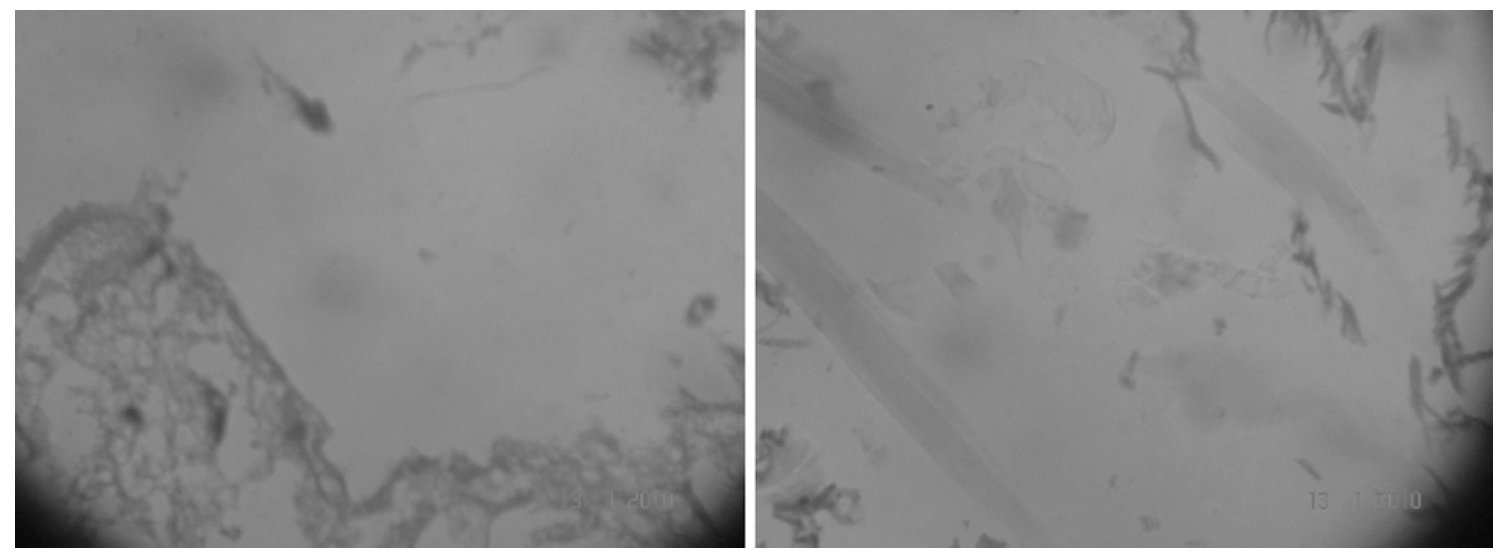

Fig. 4. Pathological examination of the cyst showing thin walled capsule in left image and flecks of keratinous material in right images. 


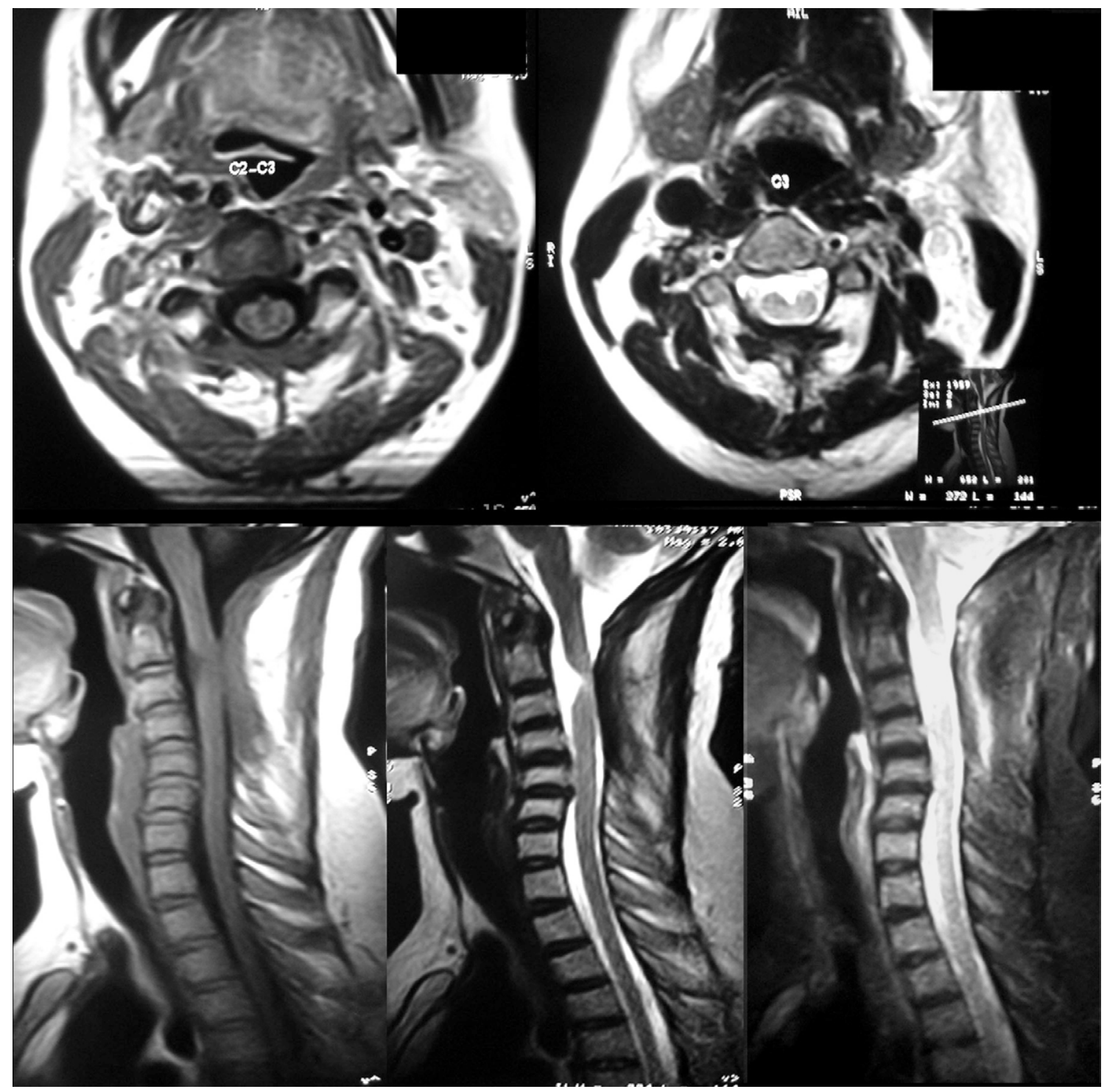

Fig. 5. Follow up magnetic resonance imaging showing complete excision of the lesion and thinning of the cord at that level.

\section{Discussion}

It has been postulated that the low incidence of epidermoids-dermoids in the cervical region is likely to be related to the embryological process of neural tube closure, which begins in the area of the lower cervical cord and proceeds rostrally and caudally [1]. These epidermoid-dermoid cysts are masses of desquamated epithelial cells and keratohyaline, encapsulated by a layer of stratified, squamous epithelial cells, usually filled with a soft, whitish yellow waxy substance with hairs and glandular secretions in addition to areas of induration [3]. MRI is the excellent imaging modality for evaluating and delineating these intradural tumors
[4]. Epidermoid-dermoid tumors can be differentiated from intrinsic glial tumors of the cord on the basis of the heterogeneity of the T1 or T2-weighted signal, lack of contrast enhancement, and signal characteristics consistent with lipid content $[1,4]$. On conventional T1W and T2W images epidermoid-dermoid lesions can have similar characteristics as of arachnoid cysts (hypointense T1- and hyperintense T2-weighted images) [3]. It has been demonstrated that the epidermoids have signal intensity similar to cerebrospinal fluid (CSF) on both T1WI and T2WI and rarely, they may be heterogenous and demonstrate high T1W signal intensity as well as peripheral rim enhancement [4]. Recently diffusion weighted imaging (DWI) of the spine has shown promise in differentiating epidermoid-dermoid cysts from 
arachnoid cysts $[3,5,6]$. In an study it was demonstrated that all spinal epidermoid-dermoid cysts showed restricted diffusion, while there was no restricted water diffusion in arachnoid cysts and concluded that DWI had no false-positive or false-negative results when correlated with final diagnosis [3]. Further it was demonstrated that epidermoid-dermoid cysts, had restricted apparent diffusion coefficient in comparison to CSF because of the squamous epithelial cells in the cyst, indicating their solid non-free-water nature and thereby slower diffusion [7]. As in present case, rarely epidermoid cyst can have clear contents [8,9], and an MRI finding can closely mimic the features of arachnoid cyst, findings not classical and is different than described in literature. Treatment of spinal epidermoids-dermoids is usually surgical but when it is tightly adhered to neural structures, and the indolent, benign nature of these lesions, to avoid unnecessary neurologic deficits, the complete removal of the cyst capsule is not recommended should not be attempted $[1-3,10]$.

\section{REFERENCES}

1. Ogden AT, Khandji AG, McCormick PC, Kaiser MG. Intramedullary inclusion cysts of the cervicothoracic junction: report of two cases in adults and review of the literature. J Neurosurg Spine 2007;7:236-42.

2. Tekkök IH. Intramedullary epidermoid cysts. J Neurosurg
Spine 2008;8:202-3.

3. Kukreja K, Manzano G, Ragheb J, Medina LS. Differentiation between pediatric spinal arachnoid and epidermoiddermoid cysts: is diffusion-weighted MRI useful? Pediatr Radiol 2007;37:556-60.

4. Bloomer CW, Ackerman A, Bhatia RG. Imaging for spine tumors and new applications. Top Magn Reson Imaging 2006;17:69-87.

5. Teksam M, Casey SO, Michel E, Benson M, Truwit CL. Intraspinal epidermoid cyst: diffusion-weighted MRI. Neuroradiology 2001;43:572-4.

6. Gottfried ON, Rao G, Anderson RC, Hedlund GL, Brockmeyer DL. Diffusion restriction of a spinal arachnoid cyst: case illustration. J Neurosurg 2005;102(4 Suppl):439.

7. Dutt SN, Mirza S, Chavda SV, Irving RM. Radiologic differentiation of intracranial epidermoids from arachnoid cysts. Otol Neurotol 2002;23:84-92.

8. Scheinfeld N. A midline cyst containing clear fluid in an infant and review of management. Pediatr Dermatol 2006;23:56-60

9. Agrawal A, Pratap A, Sinha AK, Agrawal B, Thapa A, Bajracharya T. Epidermoid cyst of anterior fontanelle with clear contents. Surg Neurol 2007;68:313-5.

10. Roux A, Mercier C, Larbrisseau A, Dube LJ, Dupuis C, Del Carpio R. Intramedullary epidermoid cysts of the spinal cord: case report. J Neurosurg 1992;76:528-33. 